\title{
Antidiabetic drug omitting, eight precepts practice and hyperglycemic problem: A case study
}

Sir,

The effect of rooted culture and belief on diabetic care is a great concern for general practitioner. In my previous report, "the relation of diabetes mellitus care to religious bound dietary pattern" is discussed. ${ }^{[1]}$ In fact, the problematic eating practices can be in either holy feast or fast. Here, I would like to present and discuss on an interesting case study of abrupt poorly controlled blood sugar in a female diabetic patient who have previously good glycemic control and relationship to the religious practice. The case is a 57 -years-old female diabetic patient who had been diagnosed for diabetes mellitus for 7 years and had a regular visit to the physician with good glycemic control. On the problematic episode, the abnormal high blood glucose $(378 \mathrm{mg} / \mathrm{dL})$ could be seen. The laboratory quality control was within normal condition and patient preparation was appropriate. The history taking shows an interesting information that the patient reported that she had been practicing Buddhism with eight precepts (a religious practice according to the Buddhist principles insists on eight percepts that include omitting of these following activities: 1) killing or injuring any animals, 2) stealing, 3) having sexual activity, 4) telling lie, 5) drinking alcoholic beverage, 6), ingesting foods after noon, 7) seeing shows or using 
perfumes and 8) sleeping on soft bed) for one month during the Buddhist lent period (she has never practiced this in the past). According to the percepts, eating after noon is prohibited but drinking is allowable. Hence, she avoided dinner but supplemented with lot of juice instead. (For breakfast and lunch, she ate and drank normally) Additionally, she omitted anti-diabetic drug because she considered taking drugs is strictly prohibited according to the percepts as eating anything after noon or dinner is not allowed. This case is an interesting story. Generally, abrupt loss of glycemic control is a common situation in management of diabetic patients and to manage this problem, detailed history taking is essential.In fact, the well-known holy fast, Ramadan, is widely described cause of difficult in glycemic control. ${ }^{[2]}$ The patient with got the problem of hypoglycemia. ${ }^{[2]}$ However, the present case seems typical. Since the holy fast in this case is daily practice for a specific period with a special fast on only "solid food", the problem of hyperglycemia can be identified in the present case. As a suggestion, to manage any diabetic patients with problem of glycemic control, diabetes care professionals should enquire about fasts/nature of fasts/etc. carefully.
To successfully manage the case, cultural competence is needed, as this case shows.

Viroj Wiwanitkit

Visiting Professor, Faculty of Medicine, University of Nis, Serbia

Corresponding Author: Prof. Viroj Wiwanitkit, Wiwanitkit House, Bangkhae, Bangkok - 10160 , Thailand. E-mail:wviroj@yahoo.com

\section{REFERENCES}

1. Wiwanitkit V. Diabetic care and religious bound dietary pattern. J Soc Health Diabetes 2013;1:94-5.

2. Zantar A, Azzoug S, Belhimer F, Chentli F. Diabetes and Ramadan. Presse Med 2012;41:1084-8.

\begin{tabular}{|l|l|}
\hline \multicolumn{2}{|c|}{ Access this article online } \\
\hline Quick Response Code: & Website: \\
\hline & www.joshd.net \\
\hline & \\
\hline
\end{tabular}

